\title{
Femoral notch cleaning in anterior cruciate ligament reconstruction: a new instrument
}

\author{
Miroslav Z. Milankov ${ }^{1}$, Natasa Miljkovic ${ }^{2}$, Zoran Gojkovic ${ }^{1}$ \\ ${ }^{1}$ Department of Orthopaedic Surgery and Traumatology, Clinical Centre Vojvodina, Medical School, University of Novi Sad, Novi \\ Sad, Serbia; \\ ${ }^{2}$ Plastic Surgery Research Laboratory, University of Pittsburgh, Pittsburgh, United State. \\ Email: milankom@eunet.rs; miljkovicnd@upmc.edu
}

Received 30 November 2009; revised 28 December 2009; accepted 2 January 2010.

\begin{abstract}
This article describes using of this new, improvised instrument that enables better removal of ACL remnants, increased visibility of the intercondylar notch, precise location of the femoral tunnel and by doing this shor- tens the operative time. Also, the use of this instrument is cost beneficial, because it decreases the number of disposable shaver tips used and can decrease the cost of the procedure which is important for countries with poor economies.
\end{abstract}

Keywords: Anterior Cruciate Reconstruction; Femoral Notch Cleaning; Improvised Instrument

\section{INTRODUCTION}

Optimal anatomical replacement of anterior cruciate ligament (ACL) is essential for achieving knee stability. The most frequent surgical error causing ACL reconstruction failure is anterior and more vertical femoral tunnel placement $[1,2]$. One potential reason for this is failure to accurately identify the true over-the-back position on the femur in the posterior aspect of the notch. Debridement of soft tissue in the notch using radiofrequency energy, shaver or different type of rasps is necessary in ACL reconstruction of the knee. In order to simplify and speed up the removal of the ACL remnants at the femoral insertion, a new, improvised instrument for ligament remnants removal has been developed.

\section{TECHNICAL NOTE}

An all-inside arthroscopical procedure for ACL reconstruction was used. After the autograft had been harvested, arthroscopic examination of the knee was performed. The ACL remnants in close contact with the PCL were removed using a shaver (Figure 1). Using a medial portal, a special instrument for the removal of the ACL ligament remnants at the femoral insertion, was introduced into the knee. The instrument comprised of a $10 \mathrm{~cm}$ long, headless, cancellous screw with 9-10th-reads at its distal end and a proximal end fitted to a holder (Figure 2). The instrument was placed in close contact with the superior and lateral part of the intercondylar roof and by moving it from the anterior to posterior, the ACL remnants were removed from the intercondylar roof and medial part of the lateral femoral condyle. The small rest of the remnants were removed using a shaver (Figure 3). Afterwards, the procedure was continued in the usual fashion.

\section{DISCUSSION}

Accurate and anatomic tunnel placements are essential to the success of reconstruction of the anterior cruciate ligament (ACL). On the femoral side, ideal tunnel placement in the sagittal plane is in the posterior quartile [3]. In the coronal plane, the common goal is to place the tunnel between the 1 and 2 o'clock position on the left and between 10 and 11 o'clock position on the right [4].

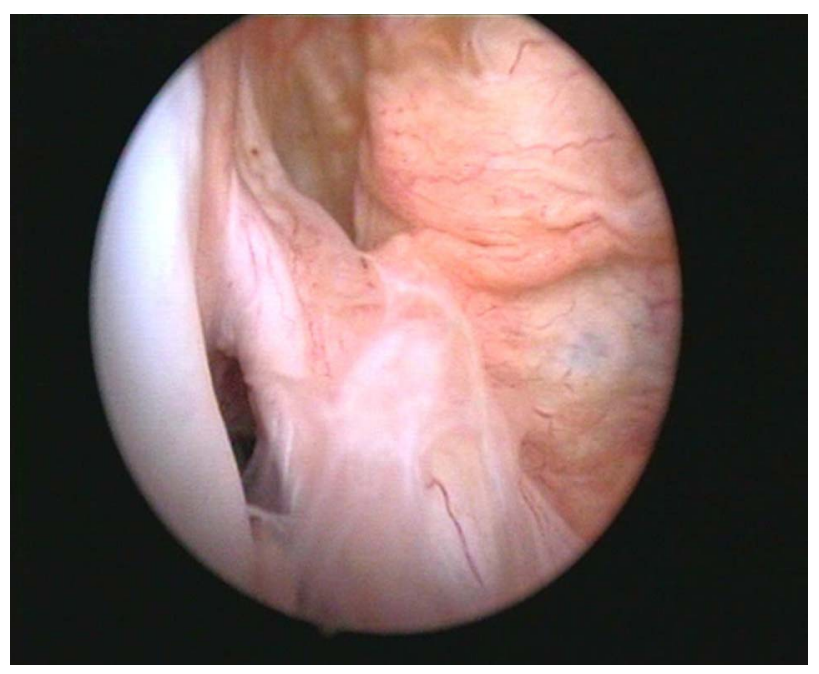

Figure 1. Arthroscopic view of the right knee from the anterolateral portal, ACL remnants. 


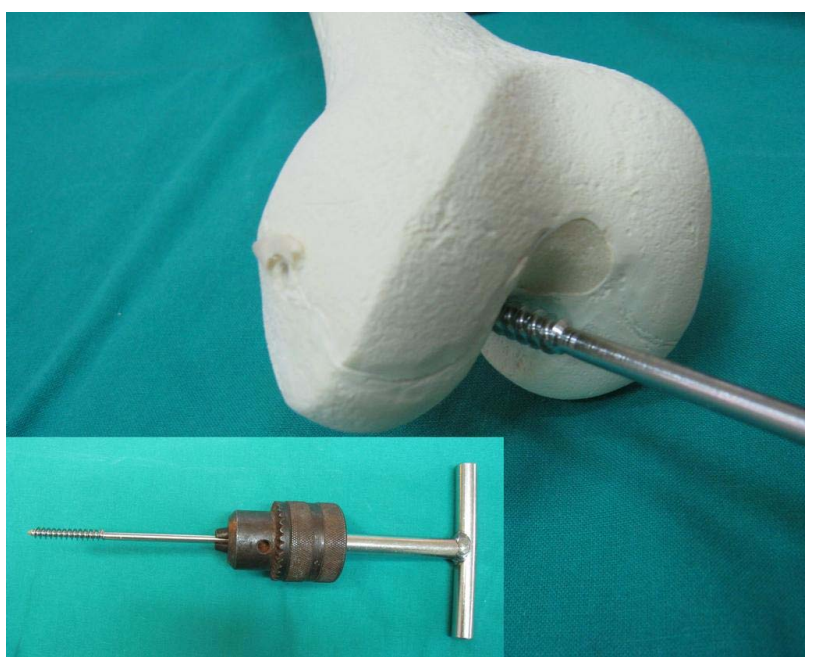

Figure 2. New, improvised instrument for ACL remnants removal. The instrument comprised of a $10 \mathrm{~cm}$ long, headless, cancellous screw with 9-10 threads at its distal end and a proximal end fitted to a holder.

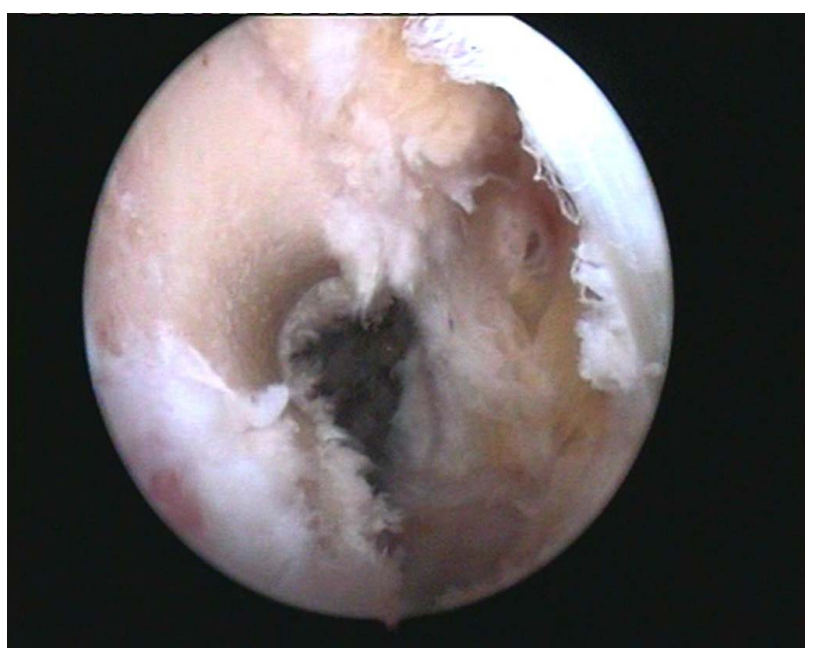

Figure 3. Arthroscopic view of the right knee from the anterolateral portal after using instrument. ACL remnants were removed from the intercondylar roof and medial part of the lateral femoral condyle. The notch doesn't have ACL remnants.

Tunnel placement based on lateral intercondylar ridge and lateral bifurcate ridge, is a more reliable approach [5-8]. The lateral intercondylar ridge is the superior border of the ACL with the knee in $90^{\circ}$ of flexion, and the anterior border of the ACL with the knee in full extension [6,7]. The lateral bifurcate ridge separates the femoral attachment of the AM and PL bundles [6,7]. The visualization of these landmarks requires meticulous rem- nant removal. Failure to recognize these bone landmarks, leads to nonanatomic placement of the femoral tunnel and, therefore, premature failure of reconstructions. In older patients with poor bone quality, one has to be very careful not to overdo with the ACL remnant removal, since this can result in a significant bone indentation. Up till now, we have used this instrument in over 200 arthroscopic ACL reconstruction procedures without any difficulty or complications.

\section{CONCLUSIONS}

The use of this new, improvised instrument enables better removal of ACL remnants, increased visibility of the intercondylar notch and precise location of the femoral tunnel and by doing this shortens the operative time. Also, the use of this instrument is cost beneficial, because it decreases the number of disposable shaver tips used and can decrease the cost of the procedure which is important for countries with poor economies.

\section{REFERENCES}

[1] Carson, E.W., Simonian, P.T., Wickiewicz, T.L. and Warren, R.F. (1998) Revision anterior cruciate ligament reconstruction. Instructional Course Lectures, 47, 361-368.

[2] Sommer, C., Friederich, N.F. and Müller, W. (2000) Improperly placed anterior cruciate ligament grafts: Correlation between radiological parameters and clinical results. The Journal of Knee Surgery, 8, 207-213.

[3] Bernard, M., Hertel, P., Hornung, H. and Cierpinski, Th. (1997) Femoral insertion of the ACL, radiographic quadrant method. American Journal of Knee Surgery, 10, 14-22.

[4] Loh, J.C., Fukuda, Y., Tsuda, E., Steadman, R.J., Fu, F.H. and Woo, S.L.Y. (2003) Knee stability and graft function following anterior cruciate ligament reconstruction: Comparison between 11 o'clock and 10 o'clock femoral tunnel placement. Arthroscopy, 19, 297-304.

[5] Farrow, L.D., Chen, M.R., Cooperman, D.R., Victoroff, B.N. and Goodfellow, D.B. (2007) Morphology of the femoral intercondylar notch. The Journal of Bone and Joint Surgery (American), 89, 2150-2155.

[6] Ferretti, M., Ekdahl, M., Shen, W. and Fu, F.H. (2007) Osseous landmarks of the femoral attachment of the anterior cruciate ligament: An anatomic study. Arthroscopy, 23, 1218-1225.

[7] Fu, F.H. and Jordan, S.S. (2007) The lateral intercondylar ridge-a key to anatomic anterior cruciate ligament reconstruction. The Journal of Bone and Joint Surgery (American), 89, 2103-2104.

[8] Hutchinson, M.R. and Ash, S.A. (2003) Resident's ridge: Assessing the cortical thickness of the lateral wall and roof of the intercondylar notch. Arthroscopy, 19, 931-935. 